\title{
Relationship status and testosterone in North American heterosexual and non-heterosexual men and women: Cross-sectional and longitudinal data
}

\author{
Sari M. van Anders, Neil V. Watson* \\ Department of Psychology, Simon Fraser University, 8888 University Drive, Burnaby, BC, Canada V5A 1S6 \\ Received 7 December 2005; received in revised form 16 January 2006; accepted 26 January 2006
}

\author{
KEYWORDS \\ Relationship status; \\ Testosterone; \\ Gender; \\ Sex; \\ Sexual orientation
}

\begin{abstract}
Summary Previous research has found that single heterosexual (Het) men have higher salivary testosterone $(\mathrm{T})$ concentrations than partnered Het men. Here, we used both longitudinal and cross-sectional analyses to examine a more diverse population $(n=258)$ that included Het and non-heterosexual (Non-Het) women and men. Results showed that, for Het men (but not Het women) and Non-Het women (but not Non-Het men), baseline T was significantly lower in partnered than unpartnered individuals. Longitudinal analyses indicated that changes in partnered status were not associated with changes in testosterone concentrations; instead, women and men with lower $\mathrm{T}$ at baseline were significantly more likely to be partnered at follow-up. These findings thus suggest that partnered status is associated with stable, trait-level $T$ values, rather than current state. Furthermore, the observed effect is limited to individuals (male or female) who are oriented toward female partners. The results are discussed in terms of evolutionary trade-offs between single and multiple partners, and the possibility of female choice and/or disinterest.

(c) 2006 Elsevier Ltd. All rights reserved.
\end{abstract}

\section{Introduction}

Previous studies have examined how testosterone (T) and relationship status are associated in heterosexual (Het) men: The earliest (Booth and Dabbs, 1993) reported that testosterone was higher in single than married male Army veterans. Although this study was cross-sectional, the

\footnotetext{
${ }^{*}$ Corresponding author. Tel.: +1604 291 3550; fax: +1 604 2913427.

E-mail address: nwatson@sfu.ca (N.V. Watson).
}

interpretation was that men with higher $\mathrm{T}$ were less likely to be married because they had more anti-social traits associated with $\mathrm{T}$ that were adaptive in the ancestral environment, but vestigial and maladaptive in the current one. Using a longitudinal approach, Mazur and Michalek (1998) found that male Air Force veterans in long-term marriages had lower $\mathrm{T}$ than veterans who stayed unwed or those who changed marital status from divorced to married or vice-versa. These authors also concluded that higher T men may be less suited for marriage. An additional observation that $T$ was elevated around divorce, but later returned 
to pre-divorce levels, was interpreted as evidence that events associated with divorce may stimulate changes in T secretion (Mazur and Michalek, 1998).

A different theoretical approach that draws on aspects of reproductive ecology posits an evolutionary trade-off between competitive behaviors aimed at acquiring/controlling partners and resources ('competitive' behaviors), and behaviors aimed towards bonding/intimacy with partners, infants, or others ('bond-maintenance' behaviors) (van Anders and Watson, in press). This can be contrasted with other existing nomenclature, like mate acquisition versus mate retention (e.g. Ketterson and Nolan, 1992), in which the important distinction is between a pre- and peri-resource state. Recent studies that can be interpreted from this perspective have found that partnered men, whether married or in long-term committed relationships, tend to have lower $\mathrm{T}$ than single men (Burnham et al., 2003; Gray et al., 2002, 2004a,b). In addition, two studies have found that partnered fathers tend to have lower testosterone levels, on average, than partnered men with no children (Burnham et al., 2003; Gray et al., 2006) although two other studies have not (Gray et al., 2002, 2004a). Time of day at which testing occurs appears to be an important variable: where these associations are found, they occur more frequently in studies with afternoon and evening hormone sampling rather than morning sampling (in contrast to Booth and Dabbs, 1993; Mazur and Michalek, 1998, who used only a.m. samples). Testosterone does show a diurnal rhythm with an increase during sleep and decrease during waking hours, such that highest levels are generally seen in the early morning and the lowest in the evening (Axelsson et al., 2005). It thus seems plausible that behavioral or cognitive factors over the course of the day could affect the rate of decline. In general, these authors (Gray et al., 2004b; Burnham et al., 2003) support a reciprocal relationship whereby high $\mathrm{T}$ is associated with competitive behavior and decreased likelihood of entering long-term relationships, but at the same time partnered/parenting interactions reduce $T$, thereby reducing competitive behaviors. In many species, lower $\mathrm{T}$ is associated with bond-maintenance behaviors aimed at mates and offspring (see Wynne-Edwards, 2001 for a review), while higher T is associated with competitive behaviors (e.g. Wingfield et al., 1990). This may also be true of humans (for reviews, see van Anders and Watson, in press; Archer, in press).

One notable limitation of previous studies is that they have sampled only Het men. There is no reason to suppose that relationships between partnered status and $\mathrm{T}$ levels are limited to a particular sex or sexual orientation, and indeed, a broader sample would permit a more comprehensive understanding of the population pattern and might point to possible mechanisms (e.g. physiological cues from partners, psychological states, etc.). For example, if all partnered individuals, regardless of sex or sexual orientation, exhibit lower testosterone than unpartnered individuals, that might suggest that the effect is mediated by partnered status alone and is unrelated to individual or partner sex.

Another impediment to generating hypotheses about causality or mechanisms is the cross-sectional nature of previous research. (Although Mazur and Michalek, 1998 reported longitudinal data, they unfortunately lacked men who were single and then wed, thus omitting a crucial group). In the present study, we attempted to address these issues by incorporating a longitudinal subsample. Further, our framework (competitive vs. bond-maintenance behaviors) posits that it is not being single per se that is associated with higher $\mathrm{T}$, but partnerseeking (competitive) behaviors. Single people engage in these behaviors, of course, but so do people in non-committed, non-monogamous, polyamorous (multiple or simultaneous loving relationships), or 'open' relationships. Previous research supports this notion: polygynous married men had higher $\mathrm{T}$ than monogamous married men (Gray, 2003). Therefore, we contrasted partnered with non-partnered (i.e. not-monogamously partnered and single) individuals, instead of with singles.

\section{Methods}

\subsection{Participants}

Participants were recruited in three ways: (1) from the psychology undergraduate participant pool, where they were prescreened for exogenous hormone use; (2) from the university community through posters; (3) from the Vancouver Pride Parade and the university community on-site at testing stations. Participants from the psychology pool received course credit for participation, and the others received small reimbursements.

Of the 293 participants initially involved in the study, 33 participants were excluded because of exogenous hormones use (e.g. hormonal contraceptives, hormonal treatments, drugs that affect endocrine systems), and two were excluded due to pregnancy, leaving 258 participants (127 males; 131 females) in the sample. Participants completed questions regarding sexual experience and fantasy on sexual orientation (Kinsey et al., 1948), and we imposed a dichotomy such that those scoring two or 
higher on either of the scales were categorized as non-heterosexual (Non-Het) $(n=106)$, while those scoring one or lower were categorized as Het $(n=$ 151), and one person did not respond. There were 55 Non-Het women (mean age $=28.20$ years), 75 Het women (mean age $=23.84$ years), 51 Non-Het men (mean age $=34.25$ ), and 76 Het men (mean age $=24.80$ years). In terms of education, the majority of participants had 1 year of undergraduate education $(n=112)$, followed by college/ university graduates $(n=57)$, high school graduates $(n=45)$, advanced degree holders $(n=27), 1$ year of advanced degree $(n=14)$, some high school $(n=2)$, and grade 7-9 $(n=1)$.

Participants indicated whether they were single, dating, in a long-term monogamous relationship but not living with their partner (paired), in a long-term monogamous committed relationship and living with their partner (established paired: married, common-law, cohabiting), in a non-monogamous relationship, and/or divorced/widowed. We then imposed a dichotomy such that paired and established paired individuals were classified as partnered, while those who were single, dating, or in non-monogamous relationships were classified as unpartnered.

\subsection{Materials and procedure}

All participants were tested between 1300 and $1800 \mathrm{~h}$ to control for diurnal rhythms in testosterone. At each session, participants provided a saliva sample and completed a brief questionnaire concerning their demographics and relationship status. Information about menstrual cycle was collected, but cycle was not controlled; previous research has shown that cycle effects are small (though consistent) and do not need to be controlled unless cycle is a variable of interest (Dabbs and de La Rue, 1991).

The first 100 participants were subsequently contacted once-monthly by email in order to track their relationship status. We started contacting participants about follow-up sessions at least 6 months after their baseline testing (and longer for participants tested earlier). Of the 100 , our final longitudinal sample consisted of 48 participants, after six participants were excluded for exogenous hormone use. Participants were not retested for the following reasons: a few requested an end to emails during the monthly contacts; some email addresses were no longer viable; some did not respond; some were away from the city during follow-up testing; some had scheduling conflicts. Follow-up testing involved the same procedures as baseline testing.
Saliva samples were collected in polystyrene tubes that had been pretreated with sodium azide, and frozen after collection at $-20^{\circ} \mathrm{C}$ until assay. Saliva was stimulated with the use of an inert gum (Trident cherry sugar-free). The samples were assayed for testosterone using radioimmunoassay in three batches at the Endocrine Core Lab at Yerkes National Primate Research Center, Emory University, all in duplicate, using a modified kit from Diagnostic Systems Laboratories (Webster, TX). The sensitivity was $2 \mathrm{pg} / \mathrm{mL}$ per $200 \mathrm{uL}$ duplicate, and the interassay coefficient of variation was $8.77 \%$ at $0.65 \mathrm{ng} / \mathrm{mL}$ and $6.88 \%$ at $5.06 \mathrm{ng} / \mathrm{mL}$. The intra-assay coefficient of variation was $6.54 \%$ at $98.82 \mathrm{pg} / \mathrm{mL}$.

Analyses were conducted with the statistical package for the social sciences (SPSS), v. 13.0. Three participants did not provide saliva samples, one male participant had $\mathrm{T}$ levels that were so high $(2073.27 \mathrm{pg} / \mathrm{mL})$ that they must have reflected unreported exogenous hormone usage or blood contamination or error, and one male had $\mathrm{T}$ that was three standard deviations over the mean; all were excluded from the analyses. Main effects were evaluated using the least significant difference (LSD) test after significant omnibus analyses.

\section{Results}

\subsection{Cross-sectional analyses (baseline)}

To control for error rate inflation due to multiple comparisons, we conducted an omnibus analysis of covariance (ANCOVA); which holds the family-wise error rate constant over derivative comparisons. We entered sex, sexual orientation, and partnered status as independent variables, age and month as covariates, and baseline $T$ as the dependent variable. There was a significant three-way interaction, $F(1,242)=4.31, p=0.039$, and so we continued with the contrasts of interest by sex and sexual orientation. Age was included as a covariate only when it accounted for a significant proportion of the variance.

The pattern of results can be summarized as follows: (1) Het unpartnered men had significantly higher $\mathrm{T}$ than Het partnered men, $F(1,73)=6.48$, $p=0.013$. (2) $T$ in Non-Het men did not differ significantly by partnered status, $F(1,45)=0.21$, $p=0.652$. (3) $T$ in Het women did not differ significantly by partnered status, $F(1,70)=0.88$, $p=0.351$. (4) Non-Het unpartnered women had significantly higher $\mathrm{T}$ than Non-Het partnered women, $F(1,52)=5.54, p=0.022$. Please see Fig. 1 for means and standard errors. Thus, 

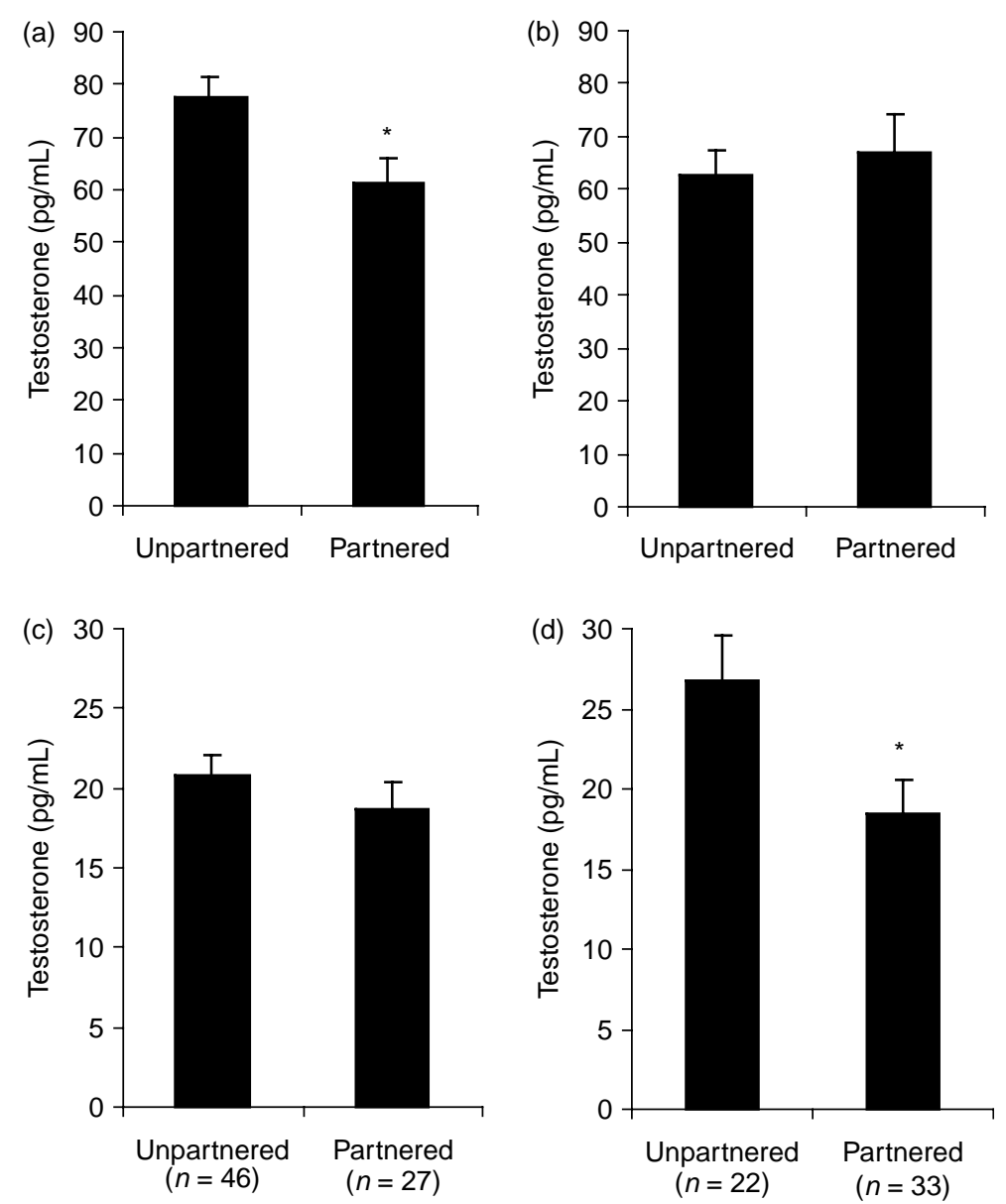

Figure 1 Means and standard errors of baseline testosterone in partnered and unpartnered individuals in (a) heterosexual men; (b) non-heterosexual men; (c) heterosexual women; (d) non-heterosexual women. *indicates a significant difference at $\alpha<0.05$.

partnered individuals had lower baseline $\mathrm{T}$ than unpartnered individuals, but only in Het men and Non-Het women. We note, here, that Non-Het women and Het men have an interest in female partners in common.

Participants were already coded as being in one of five relationship statuses (single, dating, paired, established paired, polyamorous), and we examined how these were associated with $\mathrm{T}$. First, we conducted a correlation with baseline $\mathrm{T}$ and relationship status in terms of implicit commitment (single $<$ dating $<$ paired $<$ established paired), excluding polyamoury because it did not fit conceptually in this ladder. Increased implicit commitment was significantly correlated with $\mathrm{T}$, $r(241)=-0.14, p=0.036$. With the effects of month and age partialled out, the correlation was slightly stronger, partial $r(236)=-0.19, p=0.003$. Thus, higher levels of implicit commitment were associated with lower $T$ levels.

We then conducted an ANCOVA with age and month entered as covariates when significant, relationship status (five categories), sex, and sexual orientation as the independent variables, and baseline $T$ as the dependent variable. There was a significant overall three-way interaction, $F(4$, $229)=2.56, p=0.039$. We continued with the contrasts; it should be noted that with a breakdown by sex and sexual orientation some cell sizes were small (Table 1), with an associated decrease in statistical power. Nonetheless, several patterns emerged. (1) There was a significant difference in $T$ depending on relationship status in Het men, $F(4$, $70)=2.73, p=0.036$. Men in established paired relationships had lower $T$ than singles, $p=0.006$, or polyamorous individuals, $p=0.032$. (2) There was no significant overall difference in $\mathrm{T}$ by relationship status in Non-Het men, $F(4,41)=0.93, p=0.459$. (3) There was no significant overall difference in $T$ by relationship status in Het women, $F(4,67)=$ 1.47, $p=0.222$. (4) Despite the smallest sample size, there was a trend for a significant difference in $\mathrm{T}$ by relationship status in Non-Het women, $F(4$, $49)=2.35, p=0.067$. Comparisons showed that 
Table 1 Mean baseline testosterone, standard errors in brackets, and cell numbers for heterosexual and nonheterosexual women and men by relationship status.

\begin{tabular}{llllll}
\hline & Single & Dating & Paired & Established paired & Polyamourous \\
\hline Men & & & & & \\
Heterosexual & $77.88(4.45) n=35$ & $66.22(7.79) n=11$ & $69.65(5.86) n=20$ & $49.73(8.87) n=9$ & $109.28(25.88) n=1$ \\
Non-heterosexual & $63.77(5.34) n=26$ & $59.08(15.74) n=3$ & $50.36(13.64) n=4$ & $76.45(9.11) n=9$ & $53.50(12.27) n=5$ \\
Women & & & & & \\
Heterosexual & $20.84(1.54) n=36$ & $21.37(2.79) n=11$ & $18.49(2.31) n=16$ & $15.64(3.10) n=9$ & $35.93(9.27) n=1$ \\
Non-heterosexual & $30.62(3.42) n=14$ & $24.74(6.72) n=4$ & $18.78(3.79) n=11$ & $18.26(2.77) n=21$ & $15.73(5.68) n=5$ \\
\hline
\end{tabular}

singles had higher $\mathrm{T}$ than paired, $p=0.026$, established paired, $p=0.008$, and polyamorous women, $p=0.032$. Thus, $T$ and relationship status were associated in the same groups in which the association was found with partnered status, as would be expected. Interestingly, in these groups (Het men; Non-Het women), dating individuals did not differ from any other groups, and paired (NonHet women) and established paired (Non-Het women and Het men) had lower $\mathrm{T}$ than singles. The n's for polyamourous individuals were particularly small, limiting interpretability, but this group looks promising for future research.

\subsection{Longitudinal analyses}

As described above, we collected follow-up data from 48 participants, to see if partnered status over time and $T$ were associated. We encoded Partnered status over time into four groups: stably partnered individuals (men: $n=8$; women: $n=6$ ); those who transitioned from partnered to unpartnered (no men; women: $n=3$ ); those who transitioned from unpartnered to partnered (men: $n=3$; women: $n=$ 5); and stably unpartnered individuals (men: $n=10$; women: $n=14$ ). We conducted a repeatedmeasures ANOVA with status pattern and sex as the independent variables, and testosterone at baseline and follow-up (date) as the repeated measures. Neither baseline nor follow-up month of testing, nor age, were significant covariates, and so were not included in the analyses as covariates.

There was a significant main effect of date, $F(1$, $42)=16.83, p<0.001$, such that testosterone at follow-up was significantly higher than at baseline, $p<0.001$. There was also a significant interaction between date and sex, $F(1,42)=12.09, p=0.001$, such that both sexes showed the same pattern (i.e. follow-up $>$ baseline), but men exhibited a larger increase at follow-up than women. There were no significant interactions between date and status pattern, $F(3,42)=0.114, p=0.951$, or date, sex, and status pattern, $F(2,42)=0.27, p=0.764$. See Fig. 2 for means and standard errors. Thus, there was no evidence that changes in partnered status lead to changes in $\mathrm{T}$.

The main effect of date is likely an artifact of month of testing. Though month of testing was not a significant covariate, a paired- $t$ test revealed a significant difference between season of testing at baseline and follow-up, $t(49)=-5.07, p<0.001$. Post-hoc inspection revealed that no participants received baseline testing during the months of peak $\mathrm{T}$ secretion (October, November), but $40 \%$ of follow-up sessions occurred during this time. In addition, many participants were tested during the spring and summer at baseline when $\mathrm{T}$ is low, while none were at follow-up. So, the effect of date likely reflects seasonality of testing.

\subsubsection{Between-participant effects}

There was a significant main effect of sex, $F(1$, $42)=180.27, p<0.001$, as well as a significant main effect of longitudinal partner status pattern, $F(3$, $42)=6.26, p=0.001$. The effect of status pattern was surprisingly large, $\eta^{2}=0.309$, accounting for nearly $10 \%$ of the variance in testosterone. The interaction between sex and status pattern approached significance, $F(2,42)=2.55, p=$ 0.090 , which likely was a reflection of the absence

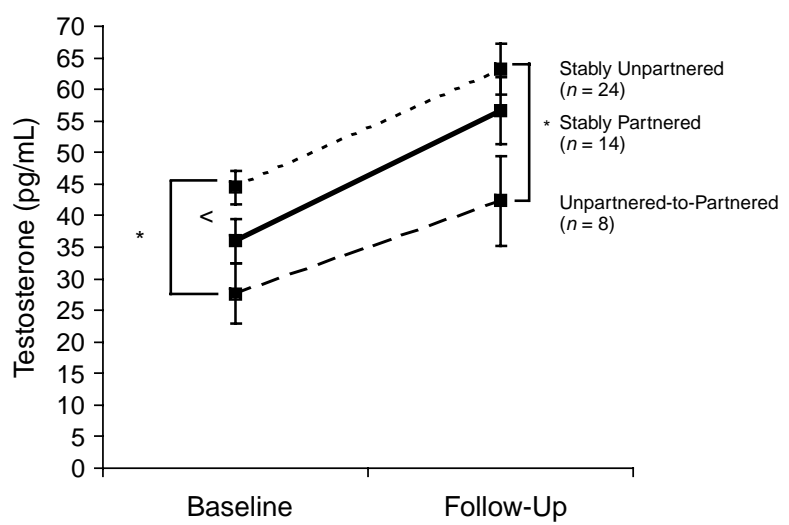

Figure 2 Means and standard errors for testosterone at baseline and follow-up in participants by their pattern of partneredness over time. *indicates a significant difference at $\alpha<0.05$ and ' $<$ ' indicates a trend towards statistical significance at $\alpha<0.10$. 


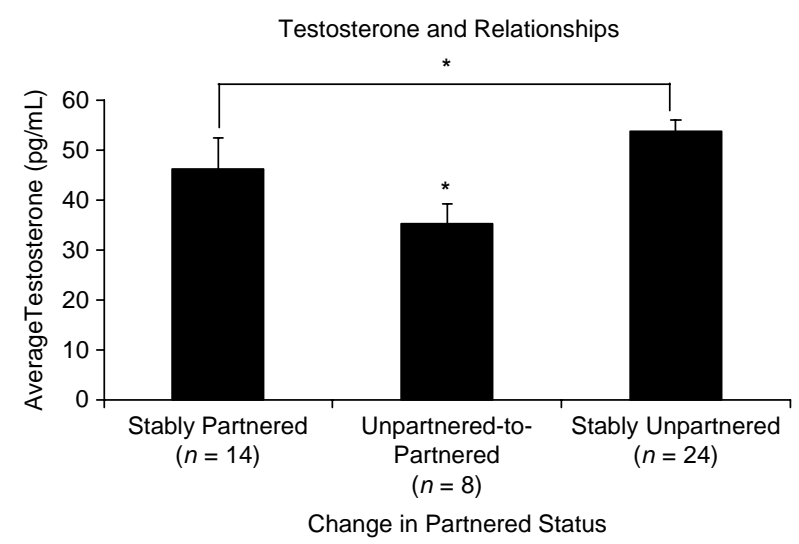

Figure 3 Means and standard errors for average testosterone (over baseline and follow-up) in participants by their pattern of partneredness over time. *indicates a significant difference at $\alpha<0.05$.

of men in one of the levels of change, since women and men showed the same pattern of $T$ in the other three levels. Examination of the main effect of status pattern on average $T$ showed that all differed significantly from each other (all $p$ 's $<0.05$; Fig. 3), though partnered-to-unpartnered is not included here due to the lack of men. Individuals who were unpartnered at baseline but partnered at follow-up had significantly lower $T$ on average than stably unpartnered individuals. This suggests that, at any one point, unpartnered individuals represent a heterogeneous group. Stably partnered individuals had significantly higher $\mathrm{T}$ than stably unpartnered individuals and significantly lower $\mathrm{T}$ than unpartnered-to-partnered individuals.

\subsubsection{Baseline testosterone and change in partnered status}

To see if baseline $T$ was associated with partnered status over time, we conducted an ANOVA with T as the dependent variable, and sex and status pattern as the independent variables. Though testosterone and partnered status are conceptually reversed here (as we are asking whether baseline T predicted future partnership status), this analysis was useful for statistical purposes. There was a significant main effect of sex, $F(1,44)=66.48, p<0.001$, and of status pattern, $F(3,44)=4.07, p=0.012$, and no significant interaction, $F(2,44)=1.22, p=0.306$. Those who were stably unpartnered had significantly higher baseline $\mathrm{T}$ than unpartnered-topartnered individuals, $p=0.003$, and marginally higher baseline $T$ than stably partnered individuals, $p=0.078$. Unpartnered-to-partnered individuals and stably partnered individuals did not significantly differ in baseline $T, p=0.102$. Please see Fig. 3 for means and standard errors. Overall, therefore, high $\mathrm{T}$ at baseline was associated with remaining unpartnered, regardless of sex.

To confirm with a more conceptually accurate analysis (in terms of independent versus dependent variables), we also calculated a median split in baseline testosterone by sex, and looked at individuals who were unpartnered at baseline in a univariate ANOVA with status pattern as the dependent variable and high/low baseline $T$ and sex as the independent variables. There was no significant main effect of sex, $F(1,28)=.691, p=$ 0.413 , nor was there a significant interaction, $F(1$, $28)=.372, p=0.120$. There was, however, a significant main effect of high/low $\mathrm{T}, F(1,28)=$ $13.08, p=0.001$, such that the unpartnered individuals with the high $\mathrm{T}$ were significantly more likely to stay unpartnered (i.e. be stably unpartnered) than the unpartnered individuals with the low $\mathrm{T}$, mirroring the above analyses.

\subsubsection{Follow-up testosterone and change in relationship status}

To see if status pattern was associated with follow-up $\mathrm{T}$, we conducted an ANOVA with follow-up $T$ as the dependent variable, and sex and status pattern as the independent variables. We did not include partnered-to-unpartnered (the category with no men). There was a significant main effect of sex, $F(1,40)=90.04, p<0.001$, and the main effect for status pattern approached significance, $F(2,40)=3.12, p=$ 0.055 . The interaction was not significant, $F(2$, $40)=1.25, p=0.298$. Stably unpartnered participants had significantly higher follow-up $T$ than unpartnered individuals who became partnered, $p=0.017$. Please see Fig. 2 for means and 
standard errors. The differences between the other groups did not approach significance.

\section{Discussion}

We examined the association between $\mathrm{T}$ levels and partnered status in non-heterosexual (Non-Het) and heterosexual (Het) women and men. As in previous studies (Burnham et al., 2003; Gray et al., 2002, 2004a,b), we found that partnered Het men have lower $\mathrm{T}$ than unpartnered Het men. However, we found that this was not specific to Het men: partnered Non-Het women have lower $\mathrm{T}$ than unpartnered Non-Het women. This effect is not apparent in Het women or Non-Het men. This suggests that the relationship between $\mathrm{T}$ and partner status is only seen in individuals who are interested in, and partner with, women.

Our longitudinal results do not support an effect of partnering on $T$ despite our expectation that partnering would lead to decreased $T$ (cf. van Anders and Watson, in press). In contrast with Mazur and Michalek (1998) who found an increase in $T$ upon marriage dissolution, we found no effect of change in partnered status on T. Our designs differed in that we did not examine divorce or marriage and we did not have men who became unpartnered as a possible comparison group. Still, in our data, becoming partnered did not lead to decreased T. However, our study differed in that the longitudinal time period was much shorter $(<1$ year) than theirs (10 years), so it is possible that longer time periods would reveal an effect. Furthermore, Mazur and Michalek (1998) reported that $\mathrm{T}$ increased around divorce, then decreased to pre-divorce levels; this might indicate that divorce does not have a permanent effect on trait levels of $\mathrm{T}$. And, since divorce generally represents a major life change that is often accompanied by debilitating consequences rather than just a change in partnered status; it may be an extreme period of stress. Gray et al. (2002) did find a negative correlation between spousal investment and evening $\mathrm{T}$; however, an alternative (and parsimonious) interpretation is that those with lower $T$ invest more. Gray et al. (2004a) found that men's T did not differ depending on days spent with their partner and children compared to those spent without their family, providing no evidence of an effect of bondmaintenance activities or partnering on $\mathrm{T}$.

One limitation of our study is the relatively small size of our longitudinal sample, though it was comparable to previous studies on relationship status and T. A replication would have a larger sample and a group of partnered individuals who become unpartnered. Conducting baseline and follow-up testing in the same season would be beneficial, since there is a large effect of seasonality on T (van Anders, Hampson and Watson, in press).

Instead of an effect of partnering on $\mathrm{T}$, our results support an influence of $T$ on partnering. Unpartnered individuals with lower $\mathrm{T}$ were more likely to become partnered than unpartnered individuals with higher $\mathrm{T}$, regardless of sex. Also, this pattern of $T$ remained stable over time. Thus, it appears that the association between testosterone and partnered status might be unidirectional and trait-level: lower $\mathrm{T}$ individuals are more likely to become and stay partnered. That the baseline population effect (partnered < unpartnered) occurs only in those who partner with women (i.e. Het men and Non-Het women) has various possible explanations. It might be advantageous for women (since they give birth), to select low T partners since low $T$ in men is associated with greater parental responsiveness (Storey et al., 2000) and better fatherchild relationships (Julian and McKenry, 1989). This remains speculative for female co-partners since $T$ and parenting in this group has received no empirical attention, though humans and other species show reproduction within long-term samesex pair bonds (Bagemihl, 1999).

It is unclear why higher $\mathrm{T}$ individuals are less likely to be partnered, but we can venture speculations. Female mate choice may play an important role, i.e. women may view higher $\mathrm{T}$ individuals as less attractive or desirable as longterm partners for reasons noted above, but potentially more attractive for short-term relationships. Thus higher $\mathrm{T}$ would not necessarily diminish reproductive opportunities even though it is associated with a decreased likelihood of partneredness. Higher $T$ individuals may be less interested in longer-term relationships as a reproductive strategy. Higher $\mathrm{T}$ is associated with decreased need for long-term commitment in women (Cashdan, 1995), as well as a higher probability of extra-marital sex (Booth and Dabbs, 1993) and increased number of sexual partners in men (Bogaert and Fisher, 1995) and women (Cashdan, 1995). In other mammalian taxa, such as voles, species that form pair bonds and engage in parental behavior have lower $T$ than those that compete for different mates (e.g. Klein and Nelson, 1997).

It is important to note that 'unpartnered' individuals form a heterogeneous category, as the $T$ levels of our unpartnered participants at baseline could be differentiated on the basis of future behavior. Previous studies support this 
interpretation of subgroups embedded within the larger category of unpartnered individuals. Gray et al. (2004) found that unpartnered men with prior relationship experience had higher $\mathrm{T}$ than unpartnered men without such experience. Conjecturally, this might be expected if the 'experienced' unpartnered group contains an over-representation of men who have serial short relationships and thus continue to be unpartnered, in contrast to 'inexperienced' unpartnered men who are unlikely to move from partner to partner.

It may be that the evolutionary trade-off between competitive and bond-maintenance behaviors within individuals is also apparent between individuals, and our data support this interpretation. We found that lower trait levels of $T$ are associated with increased likelihood of becoming partnered, and others have found that lower $\mathrm{T}$ is associated with greater parental responsiveness (Storey et al., 2000). However, changes in state levels of T can still occur regardless of one's trait level of T. In men, T dips when their partners give birth (Storey et al., 2000), and increases in response to infant stimuli (Fleming et al., 2002) perhaps in preparation for infant protection. Fathers also may have lower $\mathrm{T}$ than non-fathers (Burnham et al., 2003; Gray et al., 2006; c.f. Gray et al., 2002, 2004a), which could be interpreted that becoming a father decreases $T$. This may be conflated with infant age, and may represent a change in state $T$ in response to birth. In addition, it may be that those who become co-parents had lower trait $T$ than those who do not, even before the birth. The most parsimonious explanation, taking into account our results and the literature, is that evolutionarily influenced trade-offs occur in state levels of $T$ within individuals and trait levels of $\mathrm{T}$ between individuals.

In summary, our findings show that the pattern of lower $\mathrm{T}$ in partnered individuals reported in Het men extends to Non-Het women, and is not apparent in Non-Het men or Het women. In addition, we found no evidence that partnering modulates $T$ levels, and it is possible that the reverse relationship holds: i.e. T levels may affect the likelihood of partnering.

\section{Acknowledgements}

We would like to thank our participants, particularly our longitudinal sample for their continued participation. Thanks also to L.D. Hamilton, N. Schmidt, M. Pillay, N. Macdonald, and E. Wagner for help with data collection, and M. Schaller at the University of British Columbia for lending us lab space for extra data collection. We would also like to thank S. Gangestad, P.B. Gray, B.C. Jones, and G. van Anders for reading an earlier draft of this manuscript. Salivary assays were conducted by the Endocrine Core Assay Lab at Yerkes Primate Research Center, Emory University. A poster based on these data was presented at the annual meeting of the International Association of Sex Research in Ottawa, 2005. S.M. van Anders was supported by an NSERC PostGraduate Scholarship, a UNESCO-NSERC-L'Oreal Supplement, and a C.D. Nelson Scholarship from SFU. This research was supported by Discovery Grant 0194522 from the Natural Sciences and Engineering Research Council of Canada (NSERC) to N.V. Watson.

\section{References}

Archer, J., 2006. Testosterone and human aggression: an evaluation of the challenge hypothesis. Neurosci. Biobehav. Rev. 30, 319-345.

Axelsson, J., Ingre, M., Skerstedt, T., Holmback, U., 2005. Effects of acutely displaced sleep on testosterone. J. Clin. Endocrinol. Metab. 90, 4530-4535.

Bagemihl, B., 1999. Biological Exuberance: Animal Homosexuality and Natural Diversity. St. Martin Press, New York.

Bogaert, A.F., Fisher, W.A., 1995. Predictors of university men's number of sexual partners. J. Sex Res. 32, 119-130.

Booth, A., Dabbs Jr.., J.M., 1993. Testosterone and men's marriages. Soc. Forces 72, 463-477.

Burnham, T.C., Flynn Chapman, J., Gray, P.B., Mclntyre, M.H., Lipson, S.F., Ellison, P.T., 2003. Men in committed romantic relationships have lower testosterone. Horm. Behav. 44, 119-122.

Cashdan, E., 1995. Hormones, sex, and status in women. Horm. Behav. 29, 354-366.

Dabbs, J.M., de La Rue, D., 1991. Salivary testosterone measurements among women: relative magnitude of circadian and menstrual cycles. Horm. Res. 35, 182-184.

Fleming, A.S., Corter, C., Stallings, J., Steiner, M., 2002. Testosterone and prolactin are associated with emotional responses to infant cries in new fathers. Horm. Behav. 42, 399-413.

Gray, P.B., 2003. Marriage, parenting, and testosterone variation among Kenyan Swahili men. Am. J. Phys. Anthropol. 122, 279-286.

Gray, P.B., Kahlenberg, S.M., Barrett, E.S., Lipson, S.F., Ellison, P.T., 2002. Marriage and fatherhood are associated with lower testosterone in males. Evol. Hum. Behav. 23, 193-201.

Gray, P.B., Campbell, B.C., Marlowe, F.W., Lipson, S.F., Ellison, P.T., 2004a. Social variables predict between-subject but not day-to-day variation in the testosterone of US men. Psychoneuroendocrinology 29, 1153-1162.

Gray, P.B., Flynn Chapman, J., Burnham, T.C., McIntyre, M.H., Lipson, S.F., Ellison, P.T., 2004b. Human male pair bonding and testosterone. Hum. Nat. 15, 119-131. 
Gray, P.B., Yang, C.-F.J., Pope Jr., H.G., 2006. Fathers have lower salivary testosterone levels than unmarried men and married non-fathers in Beijing, China. Proc. R. Soc. Lond. B. Biol. Sci. 273, 333-339.

Julian, T., McKenry, P.C., 1989. Relationship of testosterone to men's family functioning at mid-life: a research note. Agressive Behav. 15, 281-289.

Ketterson, E.D., Nolan Jr., V., 1992. Hormones and life histories: an integrative approach. Am. Nat. 140, S33-S62.

Kinsey, A.C., Pomeroy, W.B., Martin, C.E., Gebhard, P.H., 1948. Sexual Behavior in the Human Male. WB Saunders, Philadelphia, PA.

Klein, S.L., Nelson, R.J., 1997. Sex differences in immunocompetence differ between two peromyscus species. Am. J. Physiol. Regul. Integr. Comp. Physiol. 273, 655-660.

Mazur, A., Michalek, J., 1998. Marriage, divorce, and male testosterone. Soc. Forces 77, 315-330.
Storey, A.E., Walsh, C.J., Quinton, R.L., WynneEdwards, K.E., 2000. Hormonal correlates of paternal responsiveness in new and expectant fathers. Evol. Hum. Behav. 21, 79-95.

van Anders, S.M., Watson, N.V., in press. Social neuroendocrinology: Effects of social contexts and behaviors on sex steroids in humans. Hum. Nat.

van Anders, S.M., Hampson, E., Watson, N.V., under review. Seasonality, Seasonality, waist-to-hip ratio, and salivary testosterone.

Wingfield, J.C., Hegner, R.E., Dufty Jr., A.M., Ball, G.F., 1990. The 'challenge hypothesis': theoretical implications for patterns of testosterone secretion, mating systems, and breeding strategies. Am. Nat. 136, 829846.

Wynne-Edwards, V.E., 2001. Hormonal changes in mammalian fathers. Horm. Behav. 40, 139-145.

$$
\begin{aligned}
& \text { Available online at www.sciencedirect.com } \\
& \text { SCiENCE }
\end{aligned}
$$

